\title{
Das Gespräch mit den Patienten ist entscheidend
}

Felicitas Witte

\footnotetext{
* Die Literaturangaben finden sich unter www.saez.ch

$\rightarrow$ Aktuelle Nummer oder

$\rightarrow$ Archiv $\rightarrow 2013 \rightarrow 33$.
}

Dr. med. Felicitas Witte

Schweizerische Ärztezeitung

EMH Schweizerischer

Ärzteverlag

Farnsburgerstrasse 8

CH-4132 Muttenz

Tel. 0614678585
Korrespondenz:

\begin{abstract}
«Medikamente wirken nicht bei Patienten, die sie nicht nehmen», sagte C. Everett Koop, Kinderchirurg und eine der einflussreichsten Personen im öffentlichen Gesundheitswesen unter Präsident Ronald Reagen in den USA, der von 1982 bis 1989 für den Präsidenten als Armee-Chirurg arbeitete. So einfach dürfe man das aber nicht sehen, sagte Bernard Vrijens, Professor für Biostatistik an der Universität Liège in Belgien und Chef der Forschungsabteilung bei MWV Healthcare, einer Verpackungsfirma, die unter anderem Medikamentenverpackungen herstellt. Vrijens berichtete kürzlich auf dem achten von Pfizer organisierten Medien-Workshop über Adherence: «Woran liegt es, dass Patienten ihre Medikamente nicht konsequent nehmen, und wie kann man das verbessern?»
\end{abstract}

\section{Adherence als Schlüssel zum Therapieerfolg}

Gemäss der Weltgesundheitsorganisation bedeutet Adherence, das «Ausmass, mit dem das Verhalten eines Patienten - sei es Medikamente, Diät und/oder Änderungen des Lebensstils, mit den Empfehlungen des Arztes korreliert» [1]*.

Das Thema Adherence werde im Arztalltag viel $\mathrm{zu}$ wenig berücksichtigt, sagte Vrijens, «dabei ist

\section{Abbildung 1}

Perfekte Adherence: Medikamenteneinnahme jeden Tag zu gleichen Uhrzeiten.

(Alle Abbildungen: Vrijens)

\section{A Patient with Perfect BID Dosing}

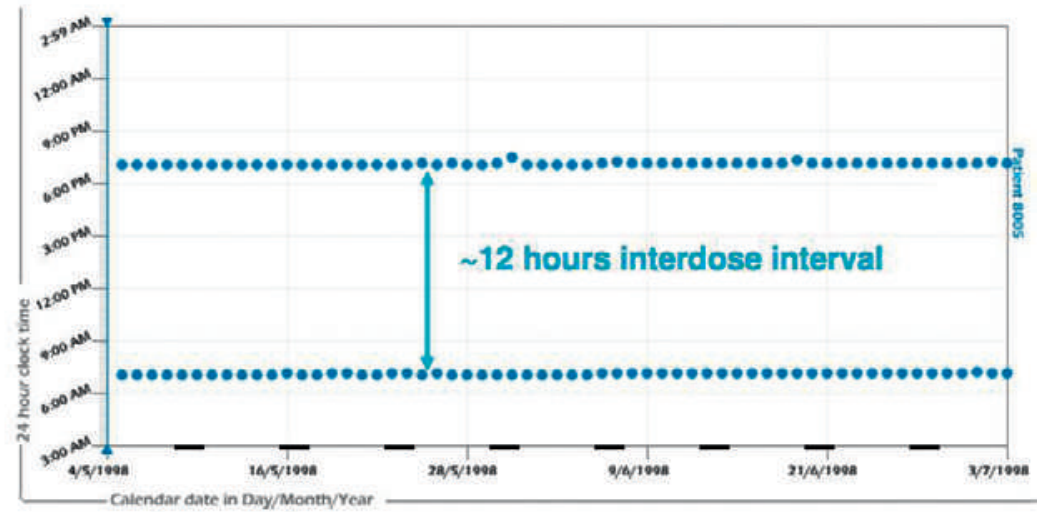

Adherence der Schlüssel, dass eine Therapie Erfolg hat». Mit computergestützten Monitoring-Systemen dokumentiert Vrijens, wann ein Patient sein Medikament genommen hat, und kann so herausfinden, was die Adherence beeinflusst. Vier Patiententypen gibt es, erklärte Vrijens am Beispiel einer Studie mit HIV-Patienten, die zweimal täglich im Abstand von 12 Stunden eine Tablette einnehmen sollten. «Einen Patienten mit der idealen Adherence gibt es fast nie», sagte Vrijens. Das ist derjenige, der jeden Tag zu exakt den gleichen Uhrzeiten seine Medikamente nimmt (Abb. 1). Typisch sei, dass die Patienten am Wochenende ausschliefen und ihre Tabletten erst am Mittag einnähmen. Der dritte Adherence-Typ nimmt anfangs die Tabletten sehr folgsam ein, dann immer unregelmässiger, bis er nach drei Monaten die Medikamente komplett absetzt (Abb. 2). Der vierte Typ hat die Tabletten schon nach der Hälfte der Studie abgesetzt.

Patienten mit verschiedenen Einnahme-Schemata scheinen auf den ersten Blick eine unterschiedliche Adherence zu haben. Doch Vrijens rechnete anhand von Beispielen aus seinen Studien vor, dass während dreier Monate alle vier Beispielpatienten 75 Prozent der verschriebenen Dosis eingenommen hatten. Die Adherence war also bei allen gleich. «Ärzte brauchen detaillierte Informationen, wann und ob jemand die Arzneimittel nimmt», sagte der Statistiker. «Bei einem gilt es dann zum Beispiel herauszufinden, warum er regelmässig die Abenddosis ausgelassen hat, beim anderen, warum er abrupt nach drei Monaten alle Tabletten abgesetzt hat.»

\section{Weniger Tabletten = bessere Adherence?}

Um zu beurteilen, wie gut die Adherence ist, analysiert Vrijens vier Aspekte: Erstens, ob der Patient überhaupt beginnt, die Medikamente einzunehmen («Initiation»). Jeder Fünfte, so berichtete der Statistiker, würde gar nicht erst die erste Pille nehmen [2]. Der zweite Aspekt ist, ob der Patient die Arznei so nimmt, wie vom Arzt verschreiben, also zum Beispiel morgens nach dem Frühstück («implementation»). Drittens dokumentiert Vrijens, ob und wann der Patient die Therapie absetzt («discontinuation»), und viertens, wie lange er die Arznei von der ersten Einnahme bis jetzt genommen hat («persistence»).

In einer Metaanalyse aus 95 Studien mit insgesamt 16907 Patienten stellte er fest, dass 40 Prozent der Patienten ihre Medikation nach 12 Monaten abgesetzt hatten [3]. Jeden Tag nahmen im Durchschnitt 15 Prozent der Patienten ihre Medika- 
Abbildung 2

Abnehmende Adherence: anfangs sehr folgsame Tabletteneinnahme, dann immer unregelmässiger, bis zum Absetzen nach drei Monaten.

\section{Worsening Regimen Execution, Then Complete Discontinuation}

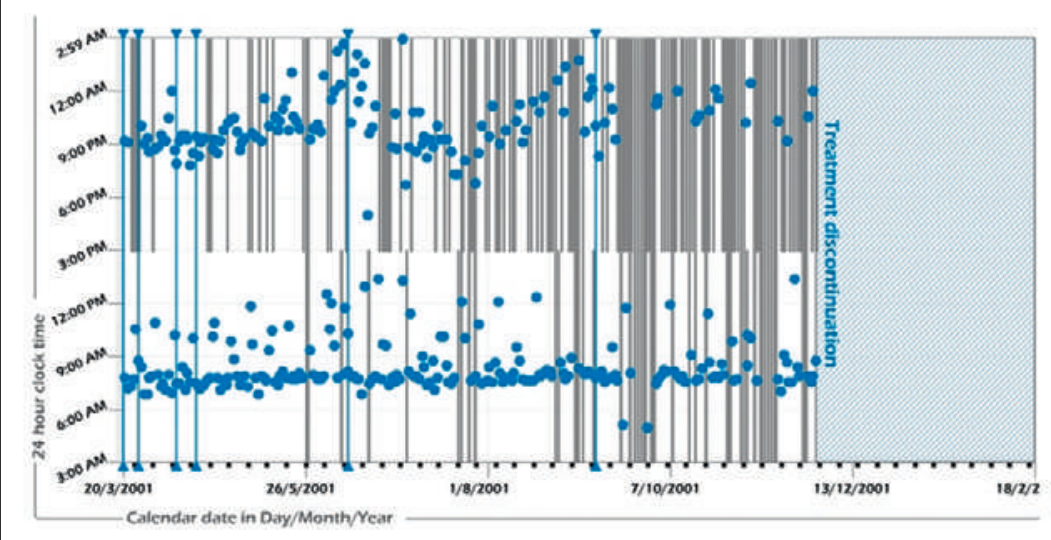

Abbildung 3

Am schlechtesten war die Adherence bei Depressionen, am besten bei Osteoporose.

\section{Persistence: \\ Time to Treatment Discontinuation}

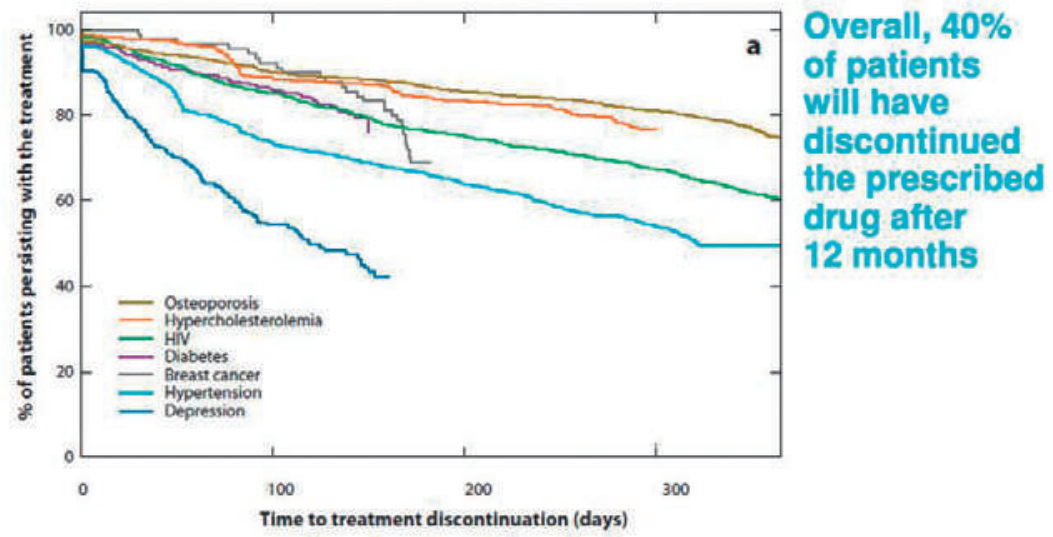

MWV

Blaschke, Osterberg, Vnjens, Urquhart, Annual Review, 2012. 11

mente nicht ein. «Die Persistence war von Krankheit zu Krankheit sehr unterschiedlich», sagte Vrijens, «das muss man im Klinik- oder Praxisalltag mit berücksichtigen.» Am schlechtesten war sie bei Depressionen, am besten bei Osteoporose (Abb. 3).

Drei Übersichtsarbeiten zeigen, dass die Adherence umso besser ist, je weniger Tabletten ein Patient einnehmen musste $[4,5,6]$. «Jeder möchte eine Einmalgabe - aber das bessert nicht immer die Adherence», sagte Vrijens. Es komme nämlich sehr auf die Pharmakokinetik und -dynamik an. «Es kommt nicht darauf an, wie viele Tabletten ein Pa- tient genommen hat, sondern wie kontinuierlich der Blutspiegel ist», erklärte der Biostatistiker. So fand der Biostatistiker heraus, dass die Wahrscheinlichkeit doppelt so gross ist, dass ein HIV-Patient mit einer Einmaldosis pro Tag seine Tablette vergisst, als dass ein Patient, der zwei- oder dreimal täglich Medikamente nimmt, diese nacheinander vergisst [7]. Um einen konstanten Blutspiegel zu erhalten, könnte die Zweimalgabe also oft besser sein als die Einmalgabe. Dass eine Tablette am Tag nicht immer die beste Lösung ist, zeigt das Beispiel der Verhütungspille: Hier kann das Vergessen einer Pille enorme Konsequenzen haben.

Am wirksamsten: mit den Patienten sprechen «Wichtig ist aber nicht nur die Konzentration des Medikamentes im Blut, sondern auch dessen pharmakokinetische Wirkung», sagte Vrijens. Wie lange ein Medikament nach einer Dosis wirkt, hängt nicht nur von der Art der Arznei ab, sondern bei manchen zudem von den pharmakodynamischen Eigenschaften. So haben viele HIV-Medikamente, vor allem die Protease-Inhibitoren, eine sehr kurze Halbwertszeit und ihre Konzentration im Plasma sinkt sehr rasch unter Werte, die nicht mehr antiviral wirken können. Ist das Dosier-Intervall - egal ob bei Einmal- oder Zweimalgabe - grösser als 36 Stunden, kann die Konzentration des Medikamentes so tief sinken, dass es nicht mehr wirkt. Bei der Einmalgabe von Proteaseinhibitoren war das in einer Studie aus Stanford bei 73\% der Patienten innerhalb von 300 Tagen mindestens einmal der Fall, bei der Zweimalgabe «nur» bei 54\% der Patienten [8]. In diesem Falle wäre es besser, die Präparate zweimal statt einmal pro Tag zu geben, weil hierbei eine vergessene Tablette weniger Konsequenzen hat.

Um die Adherence zu verbessern, gibt es eine einfache Lösung: ein ausführliches Gespräch mit dem Patienten. So zeigte Vrijens, dass sich die Adherence deutlich besserte, nachdem der Arzt dem Patienten seine Werte gezeigt und erklärt hatte [9]. Eine von der Europäischen Union gesponserte Studie betonte, dass das Gespräch mit dem Patienten und diesen über sein Einnahmeverhalten bewusst zu machen, die Adherence mehr verbesserte als andere Massnahmen wie Verhaltensänderung, Belohnungen oder psychosoziale Interventionen [10]. «Wenn wir dem Patienten seine Daten vor Augen halten, sagen viele «Huch, das sind meine Daten?» und werden sich ihres Einnahmeverhaltens bewusst», erzählt Vrijens. «Allein das bringt sie dazu, ihre Tabletten regelmässiger zu nehmen.» Klärt man Patienten darüber auf, sei das eine Dreifach-win-Situation: Der Patient profitiert, weil er besser therapiert wird, die Versicherungen, weil kein Geld für Medikamente ausgegeben wird, die dann nicht genommen werden, und nicht zuletzt die Industrie, weil weniger Studienpatienten eine Studie abbrechen. «Ärzte sollten sich Zeit nehmen und klären, woran es bei der Medikamenteneinnahme hakt», rät Vrijens. «Das müsste ihnen in Fleisch und Blut übergehen.» 


\section{Literatur}

1 WHO 2003: Adherence to long-term therapies: evidence for action.

2 Fischer MA et al. Primary Medication Non-Adherence: analysis of 195,930 electronic prescriptions. J Gen Intern Med. 2010;25(4):284-90.

3 Blaschke TF et al. Adherence to medications: insights arising from studies on the unreliable link between prescribed and actual drug dosing histories. Annu Rev Pharmacol Toxicol. 2012;52:275-301.

4 Claxton AJ et al. A systematic review of the associations between dose regimens and medication compliance. Clin Ther. 2001;23(8):1296-310.

5 Bae JP, Dobesh PP, Klepser DG, Anderson JD, Zagar AJ, McCollam PL, et al. Adherence and dosing frequency of common medications for cardiovascular patients. Am J Manag Care. 2012;18(3):139-46.

6 Coleman CI et al. Dosing frequency and medication adherence in chronic disease. J Manag Care Pharm. 2012;18(7):527-39.
7 Comté L et al. Estimation of the comparative therapeutic superiority of QD and BID dosing regimens, based on integrated analysis of dosing history data and pharmacokinetics.

J Pharmacokinet Pharmacodynam. 2007;34:549-58.

8 Blaschke TF. Variable adherence to prescribed dosing regimens for protease inhibitors: scope and outcomes. Current Opinion in HIV and AIDS. 2008;3(6):603-07.

9 Vrijens B et al. Effect of intervention through a pharmaceutical care program on patient adherence with prescribed once-daily atorvastatin. Pharmacoepidemiol Drug Saf. 2006;15(2):115-21.

10 Demonceau J et al. Identification and assessment of adherence-enhancing interventions in studies assessing medication adherence through electronically compiled drug dosing histories: a systematic literature review and meta-analysis. Drugs. 2013;73(6):545-62. 